\title{
Fetomaternal Outcome in Preterm Premature Rupture of Membrane
}

\author{
${ }^{1}$ Minakeshi Rana, ${ }^{2}$ Sharda Patra, ${ }^{3}$ Manju Puri, ${ }^{4}$ Shubha Sagar Trivedi
}

\section{ABSTRACT}

This prospective observational study was carried out to study the fetomaternal outcome in women with preterm premature rupture of membrane (PPROM) between 24 and 36 weeks of gestation.

Materials and methods: A total of 150 pregnant women between 24 and 36 weeks gestation with PROM were subjected to detailed history and examination. Each patient was followed till her delivery and fetomaternal outcome was recorded gestation-wise between 24 and 28 weeks ( $n=15), 28$ and 32 weeks $(n=30), 32$ and 34 weeks $(n=90)$, and 34 and 36 weeks $(n=15)$.

Result: The mean latency period from membrane rupture to delivery decreased from 15 days at 24 to 28 weeks, 11 days at 28 to 32 weeks to 4.4 days at 32 to 34 weeks to 2.1 days at 34 to 36 weeks. Majority of women delivered vaginally. The rate of spontaneous labor increased as the gestational age at admission increased, the difference between rate of spontaneous labor of $67 \%$ at 28 to 32 weeks and $86 \%$ at 34 to 36 weeks was statistically significant $(p=0.001)$. The indications for induction of labor were intrauterine fetal death, gross oligohydramnios, and clinical chorioamnionitis. The most common complication was clinical chorioamnionitis (6\%) and postpartum sepsis $(6 \%)$. The perinatal outcome was favorable in majority of cases and improved with the increase in gestational age at PROM. The overall perinatal mortality was $9.3 \%$.

Conclusion: Management of PPROM involves complete evaluation of risks and benefits of conservative management. Wherever possible, the treatment should be directed toward conserving the pregnancy with prophylactic use of antibiotics and steroids thereby reducing fetal-maternal morbidity and mortality. However, termination of pregnancy should be considered at the earliest suspicion of chorioamnionitis.

Keywords: Preterm premature rupture of membranes, Chorioamnionitis, Maternal outcome, Neonatal outcome.

How to cite this article: Rana M, Patra S, Puri M, Trivedi SS. Fetomaternal Outcome in Preterm Premature Rupture of Membrane. Int J Infertil Fetal Med 2014;5(1):18-21.

\section{Source of support: Nil}

Conflict of interest: None

Date of Received: 10-09-13

${ }^{1}$ Consultant, ${ }^{2}$ Associate Professor, ${ }^{3}$ Professor

${ }^{4}$ Director Professor

${ }^{1}$ Department of Obstetrics and Gynecology, Sub District Hospital Jammu, Jammu \& Kashmir, India

${ }^{2-4}$ Department of Obstetrics and Gynecology, Lady Hardinge Medical College and Smt Sucheta Kriplani Hospital, New Delhi, India

Corresponding Author: Sharda Patra, Associate Professor Department of Obstetrics and Gynecology, Lady Hardinge Medical College and Smt Sucheta Kriplani Hospital, New Delhi India, Phone: 8800166952, e-mail: shardapatra@gmail.com
Date of Acceptance: 15-03-14

Date of Publication: April 2014

\section{INTRODUCTION}

Preterm premature rupture of membrane (PPROM) is one of the most common cause of prematurity contributing to 30 to $40 \%$ of preterm births. ${ }^{1}$ There has been significant advancement in the management of PPROM, leading to an improvement in the maternal-fetal outcome. This has been made possible with use of prophylactic antibiotics and steroids. However, neonatal outcome varies from center to center depending upon the neonatal care facilities. This study was done with an aim to find out the fetomaternal outcome in PPROM managed conservatively.

\section{MATERIALS AND METHODS}

This is a prospective study carried out in the Department of Obstetrics and Gynecology in collaboration with the Department of Pathology and Pediatrics at Lady Hardinge Medical College and Smt Sucheta Kriplani Hospital, New Delhi. The study was conducted on pregnant woman attending the antenatal clinics, labor room, and maternity ward with PPROM. Pregnant women with singleton gestation in longitudinal lie with vertex presentation between 24 and 36 weeks were included in this study. Women with pregnancy-induced hypertension (PIH), antepartum hemorrhage, multiple gestation, polyhydramnios, medical disorders, including anemia, heart disease, hypertension and diabetes mellitus, and with gross congenital fetal anomalies, were excluded. Initially, each woman was admitted in the labor room for a complete maternal and fetal work-up. The baseline parameters viz demographic profile, gestational age in weeks, antenatal history, duration of premature rupture of membrane (PROM) and associated complications were noted in a prefilled performa. A complete general, physical, systemic, and obstetric examination was done initially to confirm the diagnosis of PPROM and to assess the maternal-fetal condition. Signs of overt chorioamnionitis, such as foul smelling discharge, fever, and tachycardia were looked for. A per speculum examination was done to confirm PROM and to assess the cervical parameters. Amniotic fluid was collected in a speculum or a high vaginal swab (if no leaking observed) is taken for culture and sensitivity before initiation of antibiotics. No 
per vaginam examination was done unless the woman was in labor. A baseline obstetric ultrasound was done to assess the fetal growth parameters, placenta, and the amniotic fluid volume. The woman was observed in the labor room for at least 24 hours. Those not in labor and planned for conservative treatment were transferred to the high-risk maternal ward.

Initial management included two doses of steroids $12 \mathrm{mg}$ of betamethasone 24 hours apart if pregnancy was less than 34 weeks gestation, prophylactic antibiotics injection ampicillin 1 gm, 6 hourly and injection metrogyl $500 \mathrm{mg}$ 8 hourly if PROM was $<18$ hours and injection gentamycin $80 \mathrm{mg}$ BD was added in PROM $>18$ hours. After 48 hours, oral amoxicillin $500 \mathrm{mg}$ qid was given for 5 days. Close monitoring of maternal and fetal condition was done. Antibiotics were changed according to the report of high vaginal swab (HVS) collected on admission before starting of prophylactic antibiotics. Antenatal assessment included daily vitals monitoring, obstetric examination, daily vulval pad estimation for continuing leaking, fetal growth assessment, and estimation of liquor volume was done every week by ultrasound. TLC/DLC was twice weekly and HVS was sent every week. Clinical chorioamnionitis was diagnosed if two or more of following clinical features were present. Maternal pyrexia $\left(102^{\circ} \mathrm{F}\right)$ in conjunction with uterine tenderness, purulent vaginal discharge, or fetal tachycardia. Pregnancy was terminated at 34 weeks of gestation, in women with clinical chorioamnionitis, nonreassuring fetal heart, and fetal death. If infection was identified, delivery was expedited and antibiotic stepped up. After delivery, the placenta was sent for histopathological examination and placental membranes for culture sensitivity. Baby and mother were followed for evidence of sepsis. Postpartum sepsis in the mother is recognized by the presence of fever, tachycardia, lower abdominal tenderness, leucocytosis and positive high vaginal swab culture. Newborns were closely observed for any evidence of neonatal sepsis and other compli-

Table 1: Distribution of women according to gestational age at onset of preterm premature rupture of membrane

\begin{tabular}{ll}
\hline Gestational age (weeks) & $n(\%)$ \\
\hline $24-28$ & $15(10)$ \\
$28-32$ & $30(20)$ \\
$32-34$ & $90(60)$ \\
$34-36$ & $15(10)$ \\
\hline
\end{tabular}

cations like respiratory distress syndrome (RDS), necrotizing enterocolitis, intraventricular hemorrhage, and so on.

\section{RESULTS}

Total number of deliveries conducted during the study period was 1,678 , out of this 150 women between 24 and 36 weeks had PPROM. The incidence of PPROM was $8.9 \%$. The mean age of the women was 26 years \pm 7.2 (19-36). More than $50 \%$ were primigravidae. The mean gestational age at presentation was $30.2 \pm 3.2$ weeks with majority of the women between 32 and 34 weeks (60\%) (Table 1). The median duration of leaking before admission to the hospital was 22 hours with the range between 8 and 38 hours.

The mean latency period from rupture of membranes to delivery is inversely proportional to gestation at rupture of membranes (Table 2). Lesser the gestational age at presentation longer the latency period.

Majority of women delivered vaginally. The rate of spontaneous labor increased as the gestational age at admission increased, the difference between rate of spontaneous labor of $67 \%$ at 28 to 32 weeks and $86 \%$ at 34 to 36 weeks was statistically significant $(\mathrm{p}=0.001)$ (Table 3 and Fig. 1). The indications for induction of labor were intrauterine fetal death, gross oligohydramnios, and clinical chorioamnionitis. Normal vaginal delivery occurred in almost all (100\%) at gestation $<28$ weeks and in $96 \%$ at later gestation $(>28$ 36 weeks). Cesarean section was done in $4 \%$ for indications like for fetal distress, abruptio placenta, cord prolapse, and breech presentation. On ana-lyzing the results of high vaginal swab culture taken prior to initiation, of antibiotics, it was observed that in $86 \%$ the culture was sterile. However, the culture was positive in 24 women (16\%). The most common organism grown was Escherichia Coli $(46.6 \%)$, followed by Klebsiella (33.3\%), pseudomonas (13.3\%) and coagulase negative Staphylococcus aureus (6.6\%). The most common antibiotic to which these organisms were sensitive was ampicillin (96\%), gentamicin (94\%), amikacin (96\%). Women who had growth in their vaginal swab (16\%) nearly $50 \%$ had clinical chorioamnionitis, $16 \%$ had histological chorioamnionitis, and $37 \%$ had postpartum sepsis (Table 4 ). Women who had growth in their initial HVS sample showed higher incidence of postpartum sepsis (37\%) and early neonatal sepsis $(33 \%)$, respectively. In women who had no growth in

Table 2: The gestational age at delivery and latency period with respect to gestational age at rupture of membranes

\begin{tabular}{llll}
\hline $\begin{array}{l}\text { Gestational age at admission } \\
\text { (weeks) }\end{array}$ & $\begin{array}{l}\text { Mean gest. age } \\
\text { at admission }\end{array}$ & $\begin{array}{l}\text { Gest. age at delivery } \\
\text { (mean } \pm \text { SD) (range) }\end{array}$ & $\begin{array}{l}\text { Latency period (days) } \\
\text { (mean } \pm \text { SD) (range) }\end{array}$ \\
\hline $24-28$ & $26.6 \pm 1.8$ & $28.8 \pm 5.2(26-33)$ & $15.13 \pm 10.9(6-26)$ \\
$28-32$ & $30.3 \pm 1.6$ & $32.6 \pm 1.7(29.5-34.4)$ & $11.58 \pm 9.56(3-23)$ \\
$32-34$ & $33.4 \pm 1.2$ & $34 \pm 1.02(33.1-35.2)$ & $4.92 \pm 2.88(2-9)$ \\
$34-36$ & $35 \pm 1.02$ & $35.2 \pm 0.88(34.1-36.3)$ & $2.1 \pm 0.1(1-3)$ \\
\hline
\end{tabular}

Gest: Gestational, SD: Standard deviation 


\begin{tabular}{|c|c|c|c|c|c|c|}
\hline \multirow[t]{2}{*}{ Gestational age $(n=150)$} & \multicolumn{3}{|c|}{ Spontaneous labor $(n=99)(66 \%)$} & \multicolumn{3}{|c|}{ Induced labor $(n=51)(34 \%)$} \\
\hline & Total $n(\%)$ & Vaginal & LSCS & Total n (\%) & Vaginal & LSCS \\
\hline $24-28(n=15)$ & $1(6)$ & $1(100)$ & 0 & $14(94)$ & $14(100)$ & 0 \\
\hline $28-32(n=30)$ & $20(67)$ & $20(100)$ & 0 & $10(33)$ & $8(94)$ & $2(6)$ \\
\hline $32-34(n=90)$ & $65(72)$ & $65(100)$ & 0 & $25(28)$ & $23(98)$ & $2(2)$ \\
\hline $34-36(n=15)$ & $13(86)$ & $13(100)$ & 0 & $2(14)$ & $0(0)$ & $2(100)$ \\
\hline
\end{tabular}

LSCS: Lower segment cesarean section

Table 4: Maternal complications

\begin{tabular}{ll}
\hline Maternal complications & Total $(n=150)$ \\
\hline Positive high vaginal cultures & $24(16 \%)$ \\
Clinical chorioamnionitis & $10(6.6 \%)$ \\
Histological chorioamnionitis & $4(3 \%)$ \\
Postpartum sepsis & $9(6 \%)$ \\
Retained product of conceptions (POCs) & $1(0.6 \%)$ \\
Abruptio placenta & $2(1.3 \%)$ \\
\hline
\end{tabular}

Table 5: Fetomaternal infectious complication in women who were high vaginal swab positive and high vaginal swab negative

\begin{tabular}{lll}
\hline Complications & $\begin{array}{l}\text { HVS pos. } \\
(n=24)\end{array}$ & $\begin{array}{l}\text { HVS neg. } \\
(n=126)\end{array}$ \\
\hline Clinical chorioamnionitis $(\mathrm{n}=10)$ & $7(29 \%)$ & $3(2.3 \%)$ \\
Histological chorioamnionitis $(\mathrm{n}=5)$ & $4(17 \%)$ & $1(0.7 \%)$ \\
Postpartum sepsis $(\mathrm{n}=9)$ & $9(37 \%)$ & $0 \%$ \\
Neonatal sepsis $(\mathrm{n}=8)$ & $8(33 \%)$ & $0 \%$ \\
\hline
\end{tabular}

HVS: High vaginal swab, neg.: Negative, pos.: Positive

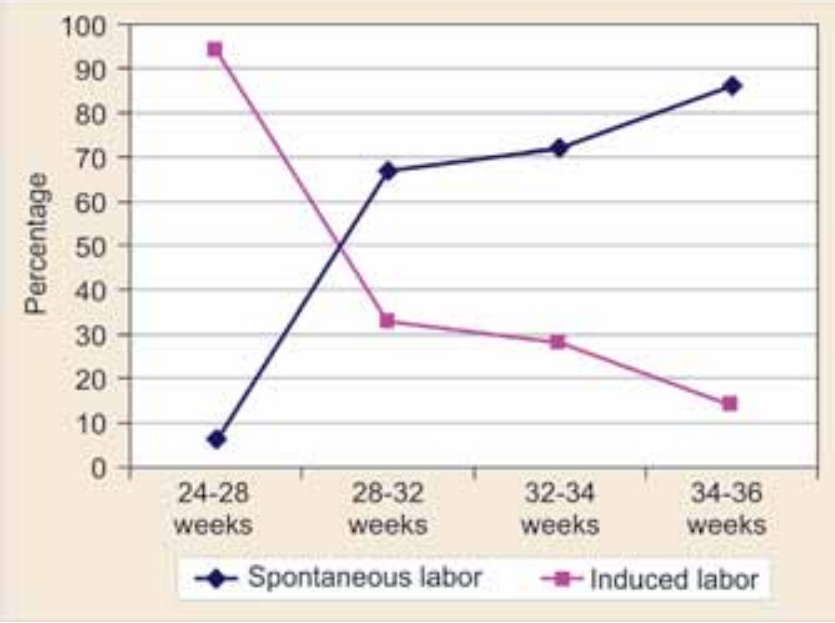

Fig. 1: Mode of delivery with respect to gestational age at presentation

their HVS, clinical chorioamnionitis was suspected in 2.3\% of women $(n=3)$ and histological chorioamnionitis in only one woman (Table 5). There were no case of postpartum sepsis and neonatal sepsis. Placental abruption was seen in two women at gestation 32 to 34 weeks. There were no other serious complications seen.

The perinatal outcome was favorable in majority of cases and improved with the increase in gestational age at PROM. The overall perinatal mortality was $9.3 \%(14 / 150)$ and it was $73 \%$ at gestation 24 to 28 weeks, $10 \%$ at 28 to 32 weeks with $0 \%$ at 32 to 36 weeks (see Table 5). The cause of death was neonatal sepsis, respiratory distress, and pulmonary hypoplasia. Babies at a gestation of $>32$ weeks had better survival rate compared with babies at less than 28 weeks $(100 \%$ vs $27 \%, \mathrm{p}=0.00)$ (Table 6$)$.

\section{DISCUSSION}

PPROM complicates up to $2 \%$ of all pregnancies and contributes to 30 to $40 \%$ of all preterm birth. ${ }^{1}$ In the current study, the prevalence of PPROM was $8.9 \%$ which is comparable to other studies reported in the literature. ${ }^{2,3}$ PROM occurring at/near term is associated with insignificant adverse effect on the mother and fetus as in 80 to $90 \%$ spontaneous onset of labor ensues. ${ }^{3}$ In women with rupture of membranes remote from term ( $<34$ weeks) prematurity as well as sepsis puts both the mother and fetus at risk. However, with use of prophylactic antibiotics and steroids, a policy of conservative management has proven beneficial to both the mother and fetus. ${ }^{4,5}$

In the present study, women presenting with PPROM from 24 to 36 weeks were managed conservatively and were followed-up till delivery. It was observed that the mean latency period decreased from 15 days at 24 to 28 weeks to 2 days at 34 to 36 weeks (see Table 2). Similar observations have been reported in the literature with latent period varying between 1 and 5 weeks. The lower the gestation the longer is the latent period. ${ }^{6}$

There was no long-term maternal sequelae. The incidence of maternal complications, especially infectious morbidity observed in the current study, is low compared to that reported in the literature. ${ }^{7-9}$ On analyzing the infectious complications in the mother and fetus, it was observed that women with positive growth in their initial vaginal samples had significantly higher rates of postpartum sepsis (37\%) and neonatal sepsis (33\%) compared with those who had no growth $(p=0.002)$. It implies relative safety of conservative management in women with PPROM with initial vaginal cultures negative. With use of antibiotics, the development of infectious complications can be further curtailed. However, women with positive culture need to be managed cautiously. Although we did not do C-reactive protein (CRP) in our patients, a recent study has shown CRP to be a highly sensitive parameter for predicting infectious morbidity in the form of chorioamnionitis. 
Table 6: Perinatal outcome

\begin{tabular}{|c|c|c|c|c|c|}
\hline Neonatal complications & $24-28(n=15)$ & $28-32(n=30)$ & $32-34(n=90)$ & $34-36(n=15)$ & Total (150) \\
\hline Neonatal sepsis & 0 & $1(3 \%)$ & $6(6 \%)$ & $1(6 \%)$ & $8(5 \%)$ \\
\hline RDS & $5(33 \%)$ & $2(6.6 \%)$ & $1(1 \%)$ & 0 & $8(5 \%)$ \\
\hline $\begin{array}{l}\text { Intraventricular } \\
\text { hemorrhage }\end{array}$ & $3(20 \%)$ & - & 0 & 0 & $3(2 \%)$ \\
\hline Pulmonary hypoplasia & $3(20 \%)$ & $1(3 \%)$ & 0 & 0 & $4(2.6 \%)$ \\
\hline \multicolumn{6}{|l|}{ Neonatal outcome } \\
\hline Stillbirths & 4 & 0 & 0 & 0 & 0 \\
\hline Early neonatal deaths & 2 & 1 & 0 & 0 & 0 \\
\hline Late neonatal deaths & 5 & 2 & 0 & 0 & 0 \\
\hline PMR & $11(73 \%)$ & $3(10 \%)$ & $0 \%$ & $0 \%$ & $14(9.3 \%)$ \\
\hline Mean birth weights (gm) & $800 \pm 100(600-1000)$ & $850 \pm 210(700-1100)$ & - & - & - \\
\hline Live births & $4(27 \%)$ & $24(80 \%)$ & $90(100 \%)$ & $15(100 \%)$ & $131(90.7 \%)$ \\
\hline Mean birth weights (gm) & $\begin{array}{l}1300 \pm 112 \\
(1200-1500)\end{array}$ & $\begin{array}{l}1750 \pm 575 \\
(1200-2300)\end{array}$ & $\begin{array}{l}2214 \pm 346 \\
(1900-2600)\end{array}$ & $\begin{array}{l}2350 \pm 380 \\
(2000-2700)\end{array}$ & - \\
\hline
\end{tabular}

PMR: Perinatal mortality rate; RDS: Respiratory distress syndrome

The neonatal outcome was favorable in majority. It was comparatively poorer in pregnancies less than 28 weeks with a perinatal mortality rate (PMR) of $73 \%$. The fetal survival increased from $80 \%$ at 28 to 32 weeks to $100 \%$ at $>32$ weeks. It is most likely a result of the use of antenatal antibiotics, corticosteroids and improved neonatal care. ${ }^{4,5}$

The incidence of neonatal sepsis was $5 \%$, a figure that is in line with numbers reported elsewhere (2-19\%). ${ }^{11-14}$ The incidence of respiratory distress syndrome and pulmonary hypoplasia in our study was also comparatively low, at 5\% and 2.6\%; however, it occurred more in gestation less than 28 weeks, a rate consis-tent with the previous reports. ${ }^{6,13,14}$ The incidence of RDS and pulmonary hypoplasia is dependent on gestational age at the time of PROM. ${ }^{6}$

In short, management of PPROM involves complete evaluation of risks and benefits of conservative management. Wherever possible, the treatment should be directed toward conserving the pregnancy with prophylactic use of antibiotics and steroids thereby reducing fetal-maternal morbidity and mortality. However, termination of pregnancy should be considered at the earliest suspicion of chorioamnionitis.

\section{REFERENCES}

1. Morris JM, Roberts CL, Crowther CA, Buchanan SL, Henderson-Smart DJ, Salkeld G. Protocol for the immediate delivery versus expectant care of woman with preterm prelabour rupture of membranes close to term (PPROM) trial. BMC Pregnancy Childbirth 2006 Mar 23;6:9. doi: 10.1186/1471-2393-6-9.

2. Smith G, Rafuse C, Anand N, Brennan B, Connors G, Crane J, Fraser W, Gratton R, Moutquin JM, Scott H, et al. Prevalence, management and outcomes of preterm prelabour rupture of the membranes of women in Canada. J Obstet Gynecol Can 2005; 27(6):547-553.
3. Tahir S, Aleem M, Aziz R. Incidence and outcome of preterm premature rupture of membranes. Pak J Med Sci 2002;18(1):26-32.

4. Kenyon SL, Taylor DJ, Tarno MW. Broad spectrum antibiotics for preterm, prelabor rupture of fetal membranes: the ORACLE I randomized trial. ORACLE Collaborative Group. Lancet 2001 Mar 31;357(9261):979-988.

5. ACOG Practice Bulletin 80. Premature rupture of membranes. Obstet and Gynecol 2007 April;109(4):1007-1019.

6. Yang LC, Taylor DR, Kaufman HH, et al. Maternal and fetal outcomes of spontaneous preterm premature rupture of membranes. JAOA 2004 Dec;104(12):537.

7. Newton ER, Prihoda TJ, Gibbs RS, et al. Logistic regression ana-lysis of risk factors for intra-amniotic infection. Obstet Gynecol 1989 Apr:73(4):571-575.

8. Soper DE, Mayhall CG. Dalton HP. Risk factors for intraamniotic infection: a prospective epidemiological study. Am J Obstet Gynecol 1989 Sep;161(3):562-566.

9. Devi A, Rani R. Premature rupture of membranes - a clinical study. J Obstet Gynecol India 1996;46:63-76.

10. Popowski T, Goffinet F, Maillaed F, Schmitz T, Leroy S, Kayen G. Maternal markers for detecting early-onset neonatal infection and chorioamnionitis in cases of premature rupture of membranes at or after 34 weeks of gestation: a two-center prospective study. BMC Pregnancy Childbirth 2011 Apr 7;11:26. doi: 10.1186/1471-2393-11-26.2011;11:26.

11. Sanyal MK, Mukherjee TN. Premature rupture of membrane; an assessment from a rural medical college of West Bengal. J Obstet Gynecol India 1990;40(4):623-628.

12. Kodkany BS, Telang MA. Premature rupture of membranes. A study of 100 cases. J Obstet Gynecol India 1991;41(4):492-496.

13. Jones G. Prelabour rupture of the membrane. Obstetrics and Gynaecology — an evidenced-based text for MRCOG. Ed. Luesley and Baker. Arnold Publisher, London 2004. Chapter 22:297-302.

14. Andrea CS, Jude PC. Preterm labor and prelabor rupture of the membranes. The Johns Hopkins Manual of Gynecology and Obstetrics. 2nd ed. Lippincot Williams \& Wilkins, USA 2000; p.123. 